\title{
AEM and FESEM Investigation of the Capacity Retention Mechanisms in Novel Composite Sulfur Copolymer Cathodes for High-Energy Density Li-S Batteries
}

\author{
J. Kim ${ }^{1}$, V.P. Oleshko ${ }^{1}$, S.D. Hudson ${ }^{1}$, C. Soles ${ }^{1}$, J.J. Griebel ${ }^{2}$, W.J. Chung ${ }^{2}$, A.G. Simmonds ${ }^{2}$, J. Pyun ${ }^{2}$ \\ ${ }^{1}$ Materials Science and Engineering Division, Material Measurement Laboratory, National Institute of \\ Standards and Technology, Gaithersburg, MD 20899 \\ ${ }^{2}$ Department of Chemistry \& Biochemistry, University of Arizona, Tucson, AZ 85721
}

Due to the growing worldwide demand for energy storage, high performance electrode materials are continuously needed to push electrochemical power sources beyond the barriers of current technologies [1]. High-energy density rechargeable batteries should be safe, environmentally friendly and reliable through thousands of charge-discharge cycles to deliver capacities $>1000 \mathrm{mAh} / \mathrm{g}$ and specific energy densities $>600 \mathrm{Wh} / \mathrm{kg}$ required for long-endurance unmanned aerial vehicle systems (UAVs) and electric vehicles (EVs) with a large distance range of action over 400 miles [1-3]. Light-weight rechargeable batteries based on the Li/elemental $\mathrm{S}$ redox couple have the highest theoretical specific capacity of 1,672 $\mathrm{mAh} / \mathrm{g}$ of active material among all solid redox couples and a specific energy of 2,567 $\mathrm{Wh} / \mathrm{kg}$, assuming complete reaction $2 \mathrm{Li}^{\mathrm{o}}+\mathrm{S}^{\mathrm{o}} \rightarrow \mathrm{Li}_{2} \mathrm{~S}[1,3]$. This can offer capacities exceeding those of conventional insertion cathodes, such as $\mathrm{LiCoO}_{2}$, by an order of magnitude. Moreover, the low cost and abundance of sulfur make Li-S batteries an attractive alternative to Li-ion batteries. Unlike insertion cathodes with layered structures, sulfur undergoes a series of topochemical transformations during cycling involving soluble polysulfides, $\mathrm{Li}_{2} \mathrm{~S}_{\mathrm{n}}(\mathrm{n}=8,6,4,3)$ and insoluble sulfides, $\mathrm{Li}_{2} \mathrm{~S}_{2}$ and $\mathrm{Li}_{2} \mathrm{~S}$. However, the utilization of the active cathode material in Li-S batteries is limited by several factors: (a) the insulating nature of sulfur, (b) losses in the form of insoluble lithium sulfides, and sometimes, (c) a rough dendritic Li anode morphology, which could result in the capacity fading and limited cycle life. Here, we demonstrate that the capacity retention in Li-S batteries, could be further improved by the use of 1,3-diisopropenylbenzene (DIB) copolymerized with molten sulfur. This new approach termed, inverse vulcanization, transforms elemental sulfur into chemically stable processable copolymers (poly(S-random-DIB)) with tunable thermomechanical properties and a high initial specific discharge capacity $(1100 \mathrm{mAh} / \mathrm{g})$, and retention of high reversible specific discharge capacity/cycle stability (823 $\mathrm{mAh} / \mathrm{g}$ at 100 cycles at rate $=\mathrm{C} / 10$ ) [4]. Cold field-emission SEM (C-FESEM) coupled with a highspeed Si-drift detector (SDD) XEDS and high-resolution FE-AEM reveal 3D multiscale architectures created within pristine and cycled composite cathodes with various contents of the electroactive copolymer (Fig. 1). The morphology, internal structures, bonding and compositional distributions of the major constituents (sulfur, poly(S-r-DIB), aggregated 30 to $60 \mathrm{~nm}$ partially disordered onion-like particles of a Timcal Super C65 conductive carbon) as well as extended pore/void mesoscale structures and their transformations under cycling have been examined by HRTEM, SAED and STEM in BF and ADF modes providing insights into mechanisms of the enhanced capacity retention in the modified Li-S cells. HAADF-STEM combined with XEDS/EELS spectral imaging (SI) (Fig. 2) allowed identifying and analyzing local distributions of the copolymer over the cathode material [5].

\section{References:}

[1] P.G. Bruce, et al, Nature Materials 11 (2012) 19. [2] http://www.bbc.co.uk/news /scienceenvironment-12074162. [3] V.P. Oleshko, et al, Microsc Microanal 15 (2009) 1398. [4] W.J. Chung, et al, Nature Chemistry 5 (2013) accepted. [5] This research was supported by NIST (Grant MML12-1053N00). 


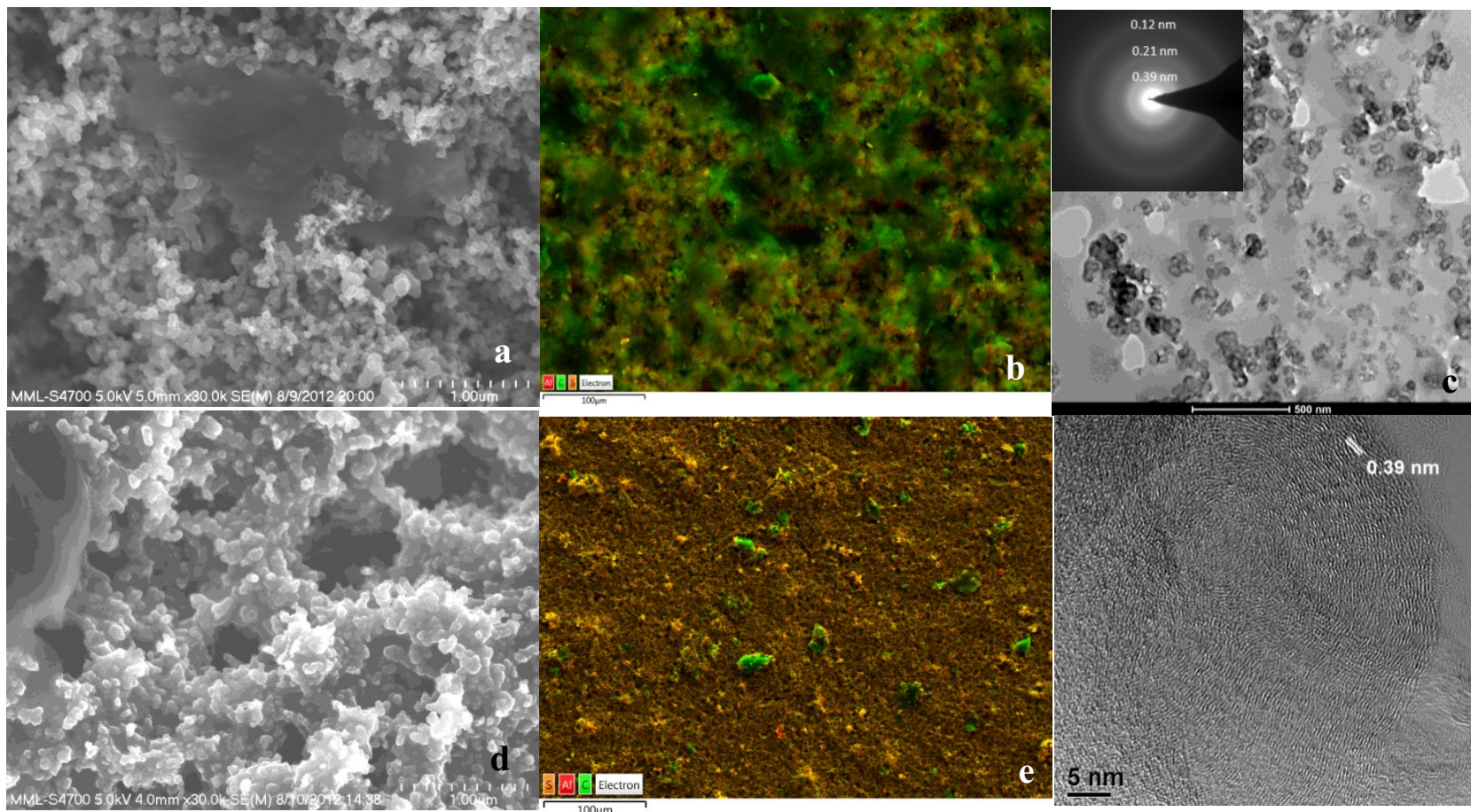

Figure 1. $(\mathrm{a}, \mathrm{b})$ Sulfur $\left(\mathrm{S}_{8}\right)$ - carbon cathode and (c-f) poly $(\mathrm{S}-r$-DIB-50-wt\% DIB) copolymer composite cathode. (a, d) C-FESEM, secondary electrons, conductive carbons with embedded sulfur particles and hierarchical porous structures; ( $b, e$ ) overlays of sulfur, aluminum (a current collector) and carbon SDD $\mathrm{X}$-ray maps and images demonstrating 50 to $150 \mu \mathrm{m}$ sulfur segregations (b), uniform sulfur distributions with no segregation and random 10 to $70 \mu \mathrm{m}$ carbon-rich compact aggregates (e), respectively; (c) TEM and SAED pattern (inset), thin section of the composite cathode displaying 30 to $60 \mathrm{~nm}$ aggregated conductive carbon particles; (f) HRTEM, an onion-like partially disordered carbon nanoparticle.

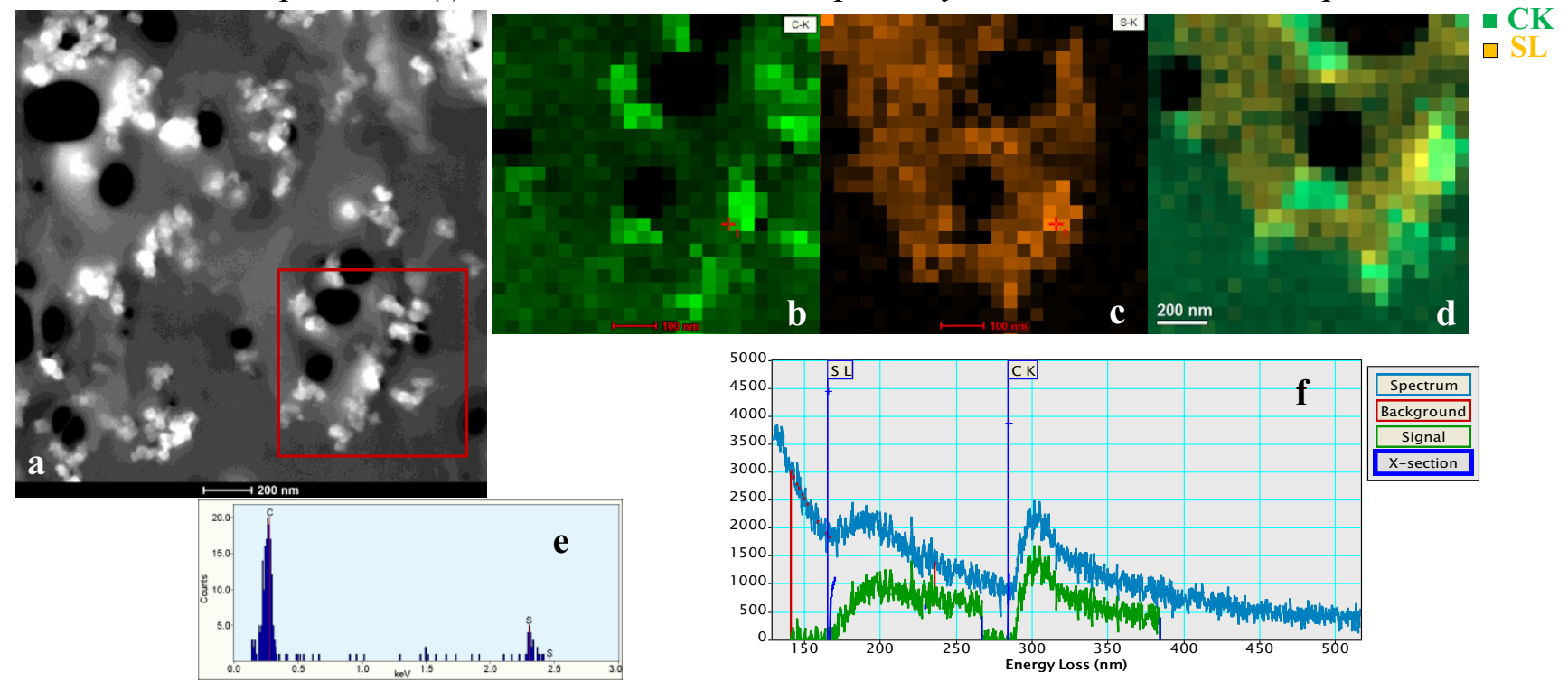

Figure 2. Poly(S- $r$-DIB-50-wt\% DIB) copolymer cathode. STEM/XEDS/EELS SI. (a) Z-contrast image revealing the copolymer distribution (medium gray), (b, c) carbon and sulfur X-ray maps; (d) overlay of carbon and sulfur EELS maps acquired from the same area marked by red square. (e) X-ray spectrum acquired in the point 1 (marked by red cross in b) and (f) EEL spectrum with a power-law assigned background, subtracted net SL- and CK-edge signals, and corresponding ionization cross-sections. 